\title{
Tratamento para escoliose pelo método de reeducação postural global (RPG) em deficientes visuais totais: série de casos
}

\author{
Treatment of scoliosis by global postural reeducation (GPR) in \\ totally visually impaired individuals: a case series
}

\author{
Graziela Morgana Silva Tavares ${ }^{1} \bowtie$, Caroline Cunha do Espírito Santo ${ }^{2}$, Patrícia Parizotto², \\ Fabiana Flores Sperandio², Gilmar Moraes Santos ${ }^{2}$ \\ ${ }^{1}$ Curso de Fisioterapia da Univesidade Federal do Pampa (Unipampa). Uruguaiana, RS. \\ 2 Programa de Pós-Graduação em Fisioterapia da Universidade do Estado de Santa Catarina (UDESC). Florianópolis, SC.
}

Este estudo foi contemplado pelo Edital PAEX 01/2009 - PROEX - Programa de Apoio à Extensão da Universidade do Estado de Santa Catarina (UDESC).

\section{RESUMO}

Objetivos: Avaliar a magnitude da escoliose em adultos jovens com deficiência visual total antes e após o tratamento por Reeducação Postural Global (RPG).

Métodos: Foram selecionados intencionalmente sujeitos com deficiência visual total, vinculados a uma instituição educacional para portadores de deficiência visual na grande Florianópolis, estado de Santa Catarina, com idade entre 18 a 40 anos, capacidade de ficar em pé de forma independente e presença de gibosidade ao exame físico. Foram excluídos indivíduos com distúrbios vestibulares, grávidas, diabéticos e amputados. Os dados radiológicos foram coletados em um serviço de referência em exames de imagem no estado de Santa Catarina antes e após oito sessões de RPG. Os dados foram analisados por meio da estatística descritiva e teste t de Student para amostra pareada.

Resultados: A amostra inicial foi composta por nove indivíduos, entretanto houve perda amostral de três sujeitos devido a desistência, suspeita de gravidez e mudança de residência, ficando a amostra final composta por seis indivíduos, quatro do gênero masculino e dois do

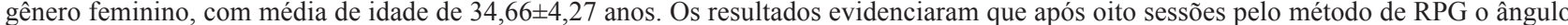
de Cobb reduziu em quatro sujeitos, manteve-se em um e aumentou em um. Entretanto, considerando-se as médias dos ângulos de Cobb pré e pós tratamento dos seis participantes, não houve diferença estatisticamente significativa. A média dos ângulos de Cobb pré-tratamento foi $8,91^{\circ} \pm 4,52^{\circ}$ e a média pós-tratamento foi $5,83^{\circ} \pm 4,52^{\circ}(\mathrm{p}=0,16)$.

Conclusões: No presente estudo, o método de RPG, após oito sessões, não alterou a magnitude média da curva escoliótica dos seis indivíduos com deficiência visual total. No entanto, na maioria dos indivíduos (quatro entre os seis) houve redução no valor do ângulo de Cobb.

DESCRITORES: postura; escoliose; pessoas com deficiência visual; raios X.

\section{ABSTRACT}

Aims: To assess scoliosis in totally visually impaired young adults by radiological examinations before and after Global Postural Reeducation (GPR).

Methods: Totally visually impaired subjects, aged 18 to 40 years, able to stand up on their own, with presence of rib hump on physical examination, attending an educational institution for the visually impaired in Florianopolis, State of Santa Catarina, Brazil, were intentionally selected. Subjects with vestibular disorders, pregnant women, diabetics, and amputees were excluded. The radiological data were collected in a reference laboratory specializing in imaging in the State of Santa Catarina before and after eight GPR sessions. The data were analyzed using descriptive statistics and the paired-sample t test.

Results: The initial sample consisted of nine individuals; however, three subjects were lost due to withdrawal, suspected pregnancy, and relocation; thus, the final sample consisted of six subjects (four males and two females) with a mean age of $34.66 \pm 4.27$ years. The results showed that after eight GPR sessions, the Cobb angle decreased in four subjects, was maintained in one, and increased in another one. However, there was no statistically significant difference in the Cobb angle $(\mathrm{p}=0.16)$ between pre-treatment $\left(8.91^{\circ} \pm 4.52^{\circ}\right)$ and post-treatment $\left(5.83^{\circ} \pm 4.52^{\circ}\right)$.

Conclusions: Global postural reeducation after eight sessions did not alter the mean magnitude of scoliosis curvature of six individuals with totally impaired vision. However, the Cobb angle decreased in most subjects (four out of six).

KEY WORDS: posture; scoliosis; visually impaired persons; X-rays. 
Abreviaturas: EVA, etileno acetato de vinila; IMC, índice de massa corporal; RPG, Reeducação Postural Global; UDESC, Universidade do Estado de Santa Catarina.

\section{INTRODUÇÃO}

De acordo com o Instituto Brasileiro de Geografia e Estatística [1], dentre os tipos de deficiência relatadas no Brasil em 2010, a deficiência visual apresentou a maior prevalência $(18,8 \%)$, representada por cerca de 35,7 milhões de pessoas deficientes visuais, sendo que 528.624 são consideradas totalmente cegas. A cegueira total, também conhecida como amaurose, é definida como a perda total da visão, ou seja, até a percepção luminosa está ausente [2,3]. Considera-se como deficiência visual congênita a que acontece antes dos três anos de idade e como adquirida a que ocorre após essa idade [4].

A deficiência visual tem como causas mais frequentes o diabetes, o descolamento de retina, os traumatismos oculares, a retinopatia da prematuridade, a catarata e o glaucoma congênitos, as degenerações retinianas e as alterações visuais corticais [5]. As deficiências visuais congênitas ou adquiridas representam a terceira razão de limitação nas atividades e mobilidades cotidianas, perdendo apenas para as cardiopatias e as artrites [6].

A perda da visão facilita o aparecimento de assimetrias posturais, caracterizadas por adaptações em diversas regiões, entre elas a cabeça (protrusão), os ombros, a pelve, o abdômen, os joelhos e a coluna vertebral, esta com inclinação posterior e aumento das curvas escolióticas [7-11]. Sanchez et al. [9] estudaram, comparativamente, a postura de adultos jovens com deficiência visual e visão normal através da biofotogrametria, e verificaram que a hiperlordose e a hipercifose torácica, assim como a escoliose, caracterizam o padrão patológico típico dos deficientes visuais. Previamente, Catanzariti et al. [7] já haviam compreendido que as ações motoras da cabeça são provocadas pela busca do melhor ângulo de visão, o que pode prejudicar ou até mesmo limitar o desenvolvimento motor esperado.

O tratamento fisioterapêutico, especificamente o método de Reeducação Postural Global (RPG), tem sido utilizado na prática clínica com relatos positivos na prevenção e recuperação de disfunções musculoesqueléticas, bem como na redução de curvas escolióticas. Durante o atendimento com a RPG, são empregadas uma ou mais posturas, que trabalham gradualmente o alongamento de músculos específicos de uma determinada cadeia (cadeia mestra anterior ou posterior), e/ou das cadeias estáticas secundárias (cadeia inspiratória, superior de ombro, anterior de braço, ântero-interna do ombro, ânterointerna do quadril e lateral do quadril). Além do sistema musculoesquelético, a RPG trabalha com o sistema sensitivo, buscando, por meio de estímulos proprioceptivos, promover a estabilidade e aperfeiçoar as reações de endireitamento dos segmentos corporais e, consequentemente, do equilíbrio [12-20].

A classificação da escoliose é obtida por meio do ângulo de Cobb, método preconizado pela Scoliosis Research Society of North America, sendo considerado método "padrão-ouro", com as curvas avaliadas mediante projeções em filmes radiográficos planos $[21,22]$. Acredita-se que a aplicação do tratamento de RPG poderia reduzir os valores do ângulo de Cobb e, consequentemente, a curva escoliótica. Toledo et al. [16], Marques [17] e Segura et al. [18] realizaram estudos com RPG em indivíduos com escoliose, com resultados que indicaram melhora na escoliose não estrutural, com redução nos valores do ângulo de Cobb. Esses estudos foram realizados com crianças e adolescentes em fase de crescimento [16-18].

Até a presente data foram encontrados poucos estudos realizados com adultos jovens com deficiência visual total para a identificação dos desvios posturais evidenciados por mensurações radiológicas [7,23]. Além disso, os estudos que realizaram intervenção em indivíduos com deficiência visual visavam a melhora do equilíbrio [24] e desenvolvimento psicomotor $[25,26]$ não se evidenciando na literatura estudos que reportassem o efeito do tratamento por RPG na escoliose desses indivíduos.

Diante do exposto, este estudo buscou avaliar a escoliose em adultos jovens portadores de deficiência visual total, mediante avaliações radiológicas antes e após aplicação de tratamento por RPG.

\section{MÉTODOS}

\section{Delineamento e seleção dos pacientes}

Este estudo caracterizou-se como sendo do tipo longitudinal, descritivo e exploratório [27]. A pesquisa foi desenvolvida de acordo com as orientações da Resolução 466/2012 do Conselho Nacional de Saúde sobre pesquisas envolvendo seres humanos, após a aprovação do comitê de ética da Universidade do Estado de Santa Catarina (protocolo no 19/2008). Todos os indivíduos foram informados sobre a natureza da pesquisa. No final da explicação, e após o esclarecimento das dúvidas e preocupações, os 
participantes assinaram o termo de consentimento livre e esclarecido com auxílio de uma régua guia.

Foram selecionados voluntários com deficiência visual total, vinculados a uma instituição educacional para pessoas portadoras de deficiência visual na grande Florianópolis, estado de Santa Catarina. Para participação no estudo os sujeitos deveriam ter idade entre 18 e 40 anos, ter capacidade de ficar em pé de forma independente (sem auxílio de órteses), apresentar deficiência visual congênita ou adquirida, já diagnosticada como total, no prontuário pertencente à associação na qual estavam devidamente cadastrados, e mostrar a presença de gibosidade [28]. Os critérios de exclusão adotados foram: presença de distúrbios vestibulares, gestação, diabetes e amputação em membros superiores ou inferiores.

\section{Procedimentos}

Foram utilizados como instrumento para execução do estudo: aparelho Silhouette VR (GE ${ }^{\circledR}$, Waukesha, Estados Unidos da América) para obtenção do raio-x panorâmico da coluna vertebral, lápis dermatográfico, régua $(50 \mathrm{~cm})$ e goniômetro de dupla haste $\left(\mathrm{CARCI}^{\circledR}\right.$, São Paulo, SP); e balança digital (Filizola ${ }^{\circledR}$, Porto Alegre, RS) escalando em quilos e intervalos de 50 gramas, para a verificação da massa corporal. A estatura foi verificada através de estadiômetro e maca de RPG.

A coleta dos dados foi feita durante os meses de agosto de 2008 a dezembro de 2009 e compreendeu três etapas: primeira etapa (pré-tratamento) - Obtenção de radiografia panorâmica da coluna vertebral. As radiografias foram feitas em um serviço de referência em exames de imagem do Estado de Santa Catarina. Os exames radiológicos foram feitos com controle do erro extrínseco (mesma hora, posição e distância, pelo mesmo profissional e, ainda, no mesmo equipamento de raio-x [29].

Segunda etapa (tratamento) - Foi realizada no Laboratório de Biomecânica da Universidade do Estado de Santa Catarina (UDESC). No primeiro momento foram verificados os dados antropométricos: peso, altura e índice de massa corporal (IMC). No segundo momento foram realizados os atendimentos pelo método RPG. Estes foram realizados de forma individual, uma vez por semana, com horário previamente marcado e duração de 50 minutos a uma hora por um período de oito semanas.

Para esse procedimento o sujeito deveria estar com os pés descalços e trajando roupa de banho e/ou ginástica. Dentre as técnicas de RPG, utilizaram-se as posturas de "rã no chão braços fechados" e "em pé no centro". Na postura "rã no chão braços fechados" é possível trabalhar principalmente a cadeia mestre anterior que possui a função estática de suspensão do corpo. Através dessa postura, a qual foi aplicada durante 35 a 40 minutos, era possível realizar as correções de coluna vertebral, tórax, respiração, ombros, cotovelos, mãos, pelve, quadril, joelhos e pés. Na postura "em pé no centro", a qual foi aplicada durante 15 a 20 minutos, é possível realizar correções da coluna vertebral, quadril, joelhos, pés, equilíbrio e esquema corporal [20], dando ao indivíduo períodos de descanso quando necessário. Os atendimentos foram conduzidos por uma fisioterapeuta com formação específica em RPG pelo método Souchard.

Para iniciar a postura "rã no chão braços fechados" o paciente foi posicionado em decúbito dorsal, com os cotovelos estendidos, ombros abduzidos a $45^{\circ}$, mãos em posição de supinação, quadris fletidos, abdução e rotação lateral, com joelhos fletidos e com as plantas dos pés tocando uma na outra. Durante a progressão da postura foi aplicada tração manual no sacro e região ocipital para auxiliar no alinhamentos da(s) curva(s) da coluna vertebral. Na região que havia a rotação e inclinação dos corpos vertebrais (escoliose), a tração foi realizada juntamente com o alinhamento da vértebra desfazendo a inclinação e derrotação para minimizar a curvatura escoliótica. Além disso, a fisioterapeuta utilizou calços de etileno acetato de vinila (EVA) na região de convexidade da curvatura escoliótica para manter a correção e alinhamento da coluna vertebral. Em membros inferiores foi realizada abdução progressiva e rotação lateral dos quadris, seguida de extensão, adução e rotação neutra de quadril e joelhos. Nos ombros foi realizada a adução progressiva dos mesmos.

Em sequência, foi realizada a postura em "pé no centro"; nesta o paciente foi posicionado com os pés em uma abertura de aproximadamente $35^{\circ}$ ou popularmente conhecida como posição de relógio (dez minutos para duas horas), com semiflexão de quadril e joelhos. A fisioterapeuta realizou então a tração, que iniciou com uma mão posicionada na base do sacro e a outra tracionando axialmente a coluna vertebral no sentido podálico para o cefálico até a base occipital, solicitando ao paciente o "crescimento", que pode ser descrito como um esforço de acompanhamento da tração do terapeuta ao alinhamento das curvaturas da coluna vertebral no eixo podálico/cefálico, como se fizesse um movimento para crescer. Os braços do paciente foram posicionados em extensão ao lado do corpo, com a mão em supinação. Durante essa postura a fisioterapeuta corrigia os segmentos corporais necessários e solicitava auxílio do paciente para o mesmo 
(é comum durante essa postura o paciente ficar com os dedos em garras; a fisioterapeuta então solicitava que o mesmo realizasse a extensão das mãos).

Terceira etapa (pós-tratamento) - Completados os atendimentos, realizou-se novamente o exame radiológico da coluna vertebral para comparar o ângulo de Cobb antes e após o tratamento.

\section{Análise dos dados}

A análise dos exames radiográficos foi realizada por um observador com mais de 10 anos de experiência em ortopedia-traumatologia, o qual não recebeu informações prévias sobre o quadro clínico dos pacientes avaliados. A avaliação foi realizada utilizando o ângulo de Cobb como parâmetro. O ângulo de Cobb foi mensurado de acordo com as informações técnicas de mensuração definidas no estudo de Vedantam et al. [30]. Em curvas maiores, o ângulo formado pelas linhas terminais é medido diretamente [31].

Posteriormente os dados foram tabulados e analisados no Programa SPSS Statistics versão 17.0. Para as variáveis quantitativas utilizou-se a estatística descritiva de média e desvio padrão. Para verificar o efeito da RPG nos desvios escolióticos foi realizada a média e desvio padrão do ângulo de Cobb dos indivíduos tanto na fase pré tratamento, bem como na fase pós tratamento. Empregou-se o teste $t$ de
Student para amostras pareadas. Foi adotado o nível de significância de $\mathrm{p} \leq 0,05$.

\section{RESULTADOS}

Dos nove voluntários que atenderam aos critérios de inclusão e iniciaram o programa, três do gênero feminino e seis do gênero masculino, houve perda amostral de três participantes, devido a desistência, suspeita de gravidez e mudança de residência. As características antropométricas dos seis que completaram as avaliações e os dados referentes ao número de curvas encontradas nas imagens radiológicas dos sujeitos antes e após o tratamento pelo método de RPG são apresentados na Tabela 1.

Não foi observada diferença estatisticamente significante entre as médias do ângulo de Cobb considerando-se os seis participantes, entre a avaliação pré-tratamento e a pós-tratamento pela RPG. $\mathrm{Na}$ avaliação pré-tratamento a média do ângulo de Cobb foi de $8,91^{\circ} \pm 4,52^{\circ}$, enquanto a média póstratamento foi de $5,83^{\circ} \pm 4,52^{\circ}(p=0,16)$. Entretanto, o comportamento não foi uniforme, pois após oito sessões de RPG, o ângulo de Cobb diminuiu em quatro entre os seis deficientes visuais $(66,6 \%)$, manteve-se em um $(16,6 \%)$ e aumentou em outro $(16,6 \%)$. Portanto, entre os seis participantes, quatro obtiveram melhora da escoliose (Tabela 1 e Figura 1).

Tabela 1. Características antropométricas dos seis participantes (adultos jovens com deficiência visual total) e número de curvas escolióticas encontradas na avaliação radiológica (incidência ântero-posterior): ângulo de Cobb antes e após oito sessões de reeducação postural global.

\begin{tabular}{|c|c|c|c|c|c|c|c|c|c|c|}
\hline Sujeito & $\begin{array}{l}\text { Idade } \\
\text { (anos) }\end{array}$ & Sexo & $\begin{array}{c}\text { Altura } \\
(\mathrm{m})\end{array}$ & $\begin{array}{l}\text { Peso } \\
(\mathrm{kg})\end{array}$ & $\begin{array}{l}\text { Tipo de } \\
\text { deficiência } \\
\text { visual }\end{array}$ & $\begin{array}{l}\text { Tempo de } \\
\text { perda visual } \\
\text { (anos) }\end{array}$ & $\begin{array}{l}\text { Número } \\
\text { de curvas }\end{array}$ & $\begin{array}{l}\text { Níveis de } \\
\text { curvas }\end{array}$ & $\begin{array}{l}\text { Ângulo de Cobb } \\
\text { pré tratamento }\end{array}$ & $\begin{array}{l}\text { Ângulo de Cobb } \\
\text { pós tratamento }\end{array}$ \\
\hline 1 & 38 & Masculino & 1.64 & 89.4 & congênita & 38 & 1 & $\mathrm{~T}$ & $20^{\circ} \mathrm{T}$ & $5^{\circ} \mathrm{T}$ \\
\hline 2 & 30 & Masculino & 1.665 & 70.8 & congênita & 30 & 2 & Te L & $8^{\circ} \mathrm{T}$ e $10^{\circ} \mathrm{L}$ & $3^{\circ} \mathrm{T}$ e $5^{\circ} \mathrm{L}$ \\
\hline 3 & 40 & Feminino & 1.49 & 65.1 & congênita & 40 & 2 & $\mathrm{~T}$ e TL & $10^{\circ} \mathrm{T}$ e $4^{\circ} \mathrm{TL}$ & $6^{\circ} \mathrm{Te} 4^{\circ} \mathrm{TL}$ \\
\hline 4 & 33 & Masculino & 1.665 & 82.5 & congênita & 33 & 2 & $\mathrm{C}$ e TL & $5^{\circ} \mathrm{Ce} 5^{\circ} \mathrm{TL}$ & $20^{\circ} \mathrm{Ce} 5^{\circ} \mathrm{TL}$ \\
\hline 5 & 37 & Feminino & 1.66 & 56.2 & congênita & 37 & 3 & $\mathrm{CT}, \mathrm{T}$ e L & $10^{\circ} \mathrm{CT}, 4^{\circ} \mathrm{T}$ e $8^{\circ} \mathrm{L}$ & $4^{\circ} \mathrm{CT}, 5^{\circ} \mathrm{T}$ e $4^{\circ} \mathrm{L}$ \\
\hline 6 & 30 & Masculino & 1.59 & 56.2 & congênita & 30 & 2 & T e L & $10^{\circ} \mathrm{Te} 13^{\circ} \mathrm{L}$ & $4^{\circ} \mathrm{T}$ e $5^{\circ} \mathrm{L}$ \\
\hline Média \pm DP & $34.66 \pm 4.27$ & & $1.62 \pm 0.07$ & $70.03 \pm 13.69$ & & $34.66 \pm 4.27$ & & & $8,91^{\circ} \pm 4,52^{\circ}$ & $5,83^{\circ} \pm 4,52^{\circ}$ \\
\hline
\end{tabular}

DP, desvio padrão; C, cervical; T, torácica; CT, cérvico-torácica; TL, tóraco-lombar; L, lombar.

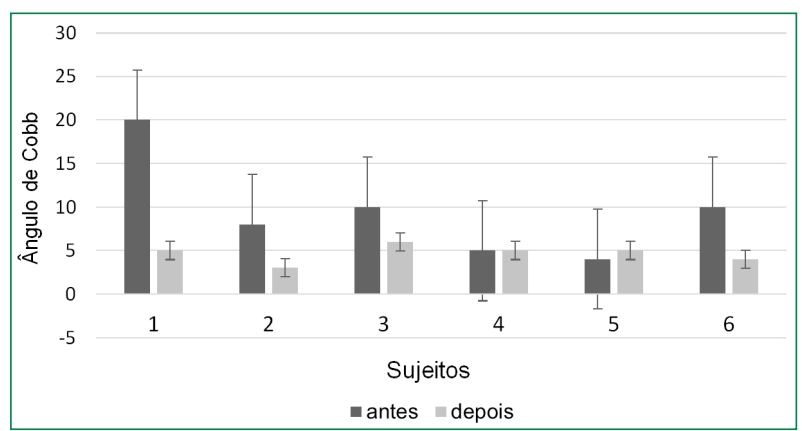

Figura 1. Valores do ângulo de Cobb antes e após o tratamento com oito sessões de reeducação postural global (RPG) em seis indivíduos com deficiência visual total. 


\section{DISCUSSÃO}

Os resultados do presente estudo, que avaliou indivíduos deficientes visuais totais com escoliose, evidenciaram que o ângulo de Cobb foi reduzido em quatro pacientes, manteve-se em um e aumentou em outro, após oito atendimentos pelo método de RPG. Entretanto, quando considerados os seis indivíduos, não houve diferença estatisticamente significativa entre a média dos ângulos de Cobb pré-tratamento e pós-tratamento.

A RPG atua diminuindo as retrações das cadeias musculares por meio da decoaptação articular realizada pelo fisioterapeuta e das contrações isométricas em posições excêntricas, que produzem alongamentos, os quais agem equilibrando as estruturas musculares e diminuindo as compensações posturais. Como consequência, diminuem as assimetrias, melhora a postura, diminuem os quadros álgicos, aumenta a flexibilidade, a amplitude de movimento e a força muscular e melhora a consciência corporal [13,20,32]. Esses fatos podem explicar a melhora de quatro indivíduos do presente estudo.

Fregnosi et al. [33] realizaram um estudo de caso com uma adolescente com escoliose lombar idiopática por meio da RPG, durante um ano, com atendimento uma vez por semana. Verificaram que houve uma diminuição da rotação da vértebra e estabilização do grau de inclinação lateral, mas para esses autores o tratamento por meio da RPG apenas estabilizou a curvatura, não sendo suficiente para reduzir a angulação da escoliose [33].

Este fato é similar ao que ocorreu no presente estudo com um dos indivíduos que não apresentou melhora e outro que apresentou leve piora. Duas hipóteses podem ser levantadas: a primeira é o fato de serem indivíduos já adultos, que haviam passado da melhor fase de intervenção, que é a fase de crescimento, tendo já uma curvatura escoliótica estruturada. A outra hipótese, ou mesmo um segundo fator que pode ter contribuído para a ausência de melhora, diz respeito aos hábitos posturais. Geralmente o deficiente visual adota uma postura inadequada e, por carecer de informação visual, mesmo o fisioterapeuta tentando orientar e corrigir a sua postura durante o atendimento, o paciente não mantém o estímulo de correção fora do atendimento. Além disso, ainda não há um consenso sobre o número mínimo de intervenções pelo método de RPG para que o indivíduo apresente melhora significativa de suas alterações posturais [15,34], em especial da escoliose [17,18,33]. Estudos futuros ainda se fazem necessários, tanto para sabermos o número de atendimentos necessários para melhora do quadro clínico, como para determinar o momento de fazer a reavaliação radiológica para verificação da melhora, manutenção ou piora do ângulo de Cobb, o que poderá determinar a manutenção, alteração ou mesmo a suspensão do tratamento pelo método de RPG.

Um fator que pode justificar a redução do ângulo de Cobb em quatro dos seis indivíduos, é que talvez estes tivessem uma melhor consciência corporal quando comparados com os outros dois sujeitos. De acordo com Toledo et al. [16] a RPG, além de considerar o indivíduo de forma global, tem como objetivo o conhecimento do próprio corpo e, assim, o aperfeiçoamento das reações de endireitamento.

Uma hipótese que também pode ser levantada para diminuição da curva escoliótica pelo método de RPG, é que este atua do centro para a parte distal do corpo, ou seja, da coluna para os membros, impedindo compensações e restabelecendo o equilíbrio muscular [16]. O tratamento conservador da escoliose idiopática é controverso $[35,36]$ e há poucos relatos na literatura mostrando a sua eficácia, principalmente em adultos jovens [37,38] e portadores de deficiência visual $[7,23,39]$.

No presente estudo foi possível observar quatro indivíduos portadores de deficiência visual que apresentavam duas curvas escolióticas, um com uma curva e um com três curvas, com uma média de diminuição do ângulo de Cobb de $-3,08^{\circ}$. De acordo com Souchard [20], as posturas em decúbitos, como a "rã no chão braços fechados" empregada no presente estudo, facilitam a correção e o reposicionamento manual por parte do fisioterapeuta, enquanto as posturas com cargas, como a "em pé ao centro", são mais ativas e proprioceptivas, permitindo assim uma melhor integração com o esquema corporal.

Cardoso et al. [40] que realizaram a análise do ângulo de Cobb em uma adolescente pré e pós 30 sessões de iso-streching, verificaram um ângulo inicial de $42^{\circ}$, e ao final do tratamento $36^{\circ}$, ocorrendo redução de $6^{\circ}$. O tratamento com iso-streching foi associado ao uso de colete toracolombossacral durante 23 horas por dia [40].

Segundo Meereis et al. [39] a privação da informação visual poderá trazer consequências importantes para o indivíduo com deficiência visual, em relação ao seu equilíbrio postural e ao desenvolvimento motor, desde a infância até a fase adulta. Gauchard et al. [41] consideram que a realização de correções posturais é benéfica para indivíduos com escoliose, pois o controle da postura é alterado significativamente. 
O sistema visual fornece informações relevantes quanto aos ambientes em que o indivíduo se encontra, além de promover a estabilidade corporal durante a locomoção, em nível local (passada) e nível global (planejamento do deslocamento) $[42,43]$. Posturas inadequadas assumidas diariamente, em conjunto com o uso assimétrico do corpo, podem provocar modificações no sistema neuromuscular, ou seja, alterações de postura $[44,45]$. A falta da informação visual pode levar o indivíduo portador de deficiência visual a compensações musculoesqueléticas no intuito de reorganizar o seu corpo, ambientá-lo ao espaço e manter o seu equilíbrio. Dentre as compensações corpóreas que ocorrem, diversos estudos evidenciaram anteriorização da cabeça, protrusão de ombros, joelhos fletidos e anteroversão pélvica [7,9]. Essas alterações posturais comprometem principalmente a cadeia mestra anterior.

Em um estudo para verificar o efeito agudo do RPG, utilizando a técnica de "rã no chão braços fechados", uma única vez, durante 40 minutos, em mulheres com o encurtamento da cadeia anterior, Rossi et al. [34] evidenciaram que a aplicação da técnica melhorou a posição da cabeça e do ombro, porém não houve alteração em outras estruturas analisadas, como o tronco, a pelve e o membro inferior. Além disso, os mesmos não realizaram exames radiográficos para verificar a presença de escoliose ou testes simples para verificar a presença de gibosidade, e efeito da técnica nesse tipo de alteração após a intervenção [34].

Morningstar et al. [36], em um estudo com 19 sujeitos portadores de escoliose (com idade entre 15 e 65 anos), avaliaram a coluna vertebral através de exame radiográfico na posição anteroposterior. Após receberem uma intervenção de manipulação da coluna vertebral em conjunto com tração e reeducação neuromuscular, em um período de quatro a seis semanas, foi realizada nova avaliação. De acordo com os resultados radiográficos e valores do ângulo de Cobb pós-intervenção, houve redução desse ângulo, com uma média geral de $17^{\circ}$.

Um estudo de triagem para escoliose comparou uma amostra de 75 crianças com deficiência visual com um grupo saudável de 728 crianças. A escoliose foi avaliada através da flexão de tronco em conjunto com uma análise fotográfica pela topografia de Moiré. Os resultados mostraram maior prevalência de gibosidade clínica nas crianças com baixa visão em comparação ao grupo sem deficiência visual (41,3\% e $13 \%$ respectivamente; $p<0,0001)$. Com isso, os autores concluíram que um sistema visual ou oculomotor deficiente pode ocasionar deformidades de tronco, mas com exceção de crianças com curvatura severa, nenhum tratamento corretivo é indicado, pois estas alterações resultam, provavelmente, de uma postura anormal da cabeça para auxiliar na função visual [7]. Sanchez et al. [9] salientam que o desenvolvimento de mecanismos sensoriais de uma criança com o sistema visual normal ocorre facilmente, ao contrário do que acontece com crianças deficientes visuais, que apresentam dificuldades no desenvolvimento do equilíbrio, coordenação motora, agilidade e mobilidade, podendo ocasionar assimetrias compensatórias [9].

O presente estudo apresentou como limitações uma amostra pequena, o curto período de acompanhamento e a falta de um grupo controle que fosse submetido a outro tipo de intervenção ou simplesmente não recebesse intervenção. Estes resultados evidenciam que o método de RPG, após oito sessões de atendimento, não alterou significativamente a magnitude do ângulo de Cobb da curva escoliótica em indivíduos adultos com deficiência visual total, quando considerada a média entre os seis indivíduos avaliados. No entanto, deve ser salientado que na maioria dos sujeitos (quatro entre os seis) houve redução na medida do ângulo de Cobb.

\section{REFERÊNCIAS}

1. Instituto Brasileiro de Geografia e Estatística. Censo demográfico 2010: características gerais da população, religião e pessoas com deficiência [Internet]. Rio de Janeiro: IBGE; 2010 [cited 2015 Sept 10]. Available from: http://biblioteca.ibge.gov.br/index.php/bibliotecacatalogo? view $=$ detalhes \&id $=794$

2. Taleb A, de Faria MAR, Ávila M, Mello PAA. As condições de saúde ocular no Brasil - 2012 [Internet]. São Paulo; 2012 [cited 2015 Sept 12]. Available from: http://www.cbo.com.br/novo/medico/pdf/01-cegueira.pdf

3. World Health Organization - WHO. Change the Definition of Blindness [Internet]. [cited 2015 Sept 15]. Available from: http://www.who. int/blindness/Change $\% 20$ the $\% 20$ Definition $\% 20$ of $\% 20$ Blindness.pdf?ua $=1$

4. Mosquera C. Educação física para deficientes visuais. Rio de Janeiro: Sprint; 2000.

5. Hoyt CS, Good WV. The many challenges of childhood blindness. Br J Ophthalmol. 2001 Oct;85(10):1145-6. http://dx.doi.org/10.1136/ bjo.85.10.1145

6. Cohen H. Neurociência para fisioterapeutas: incluindo correlações clínicas. 2a ed. São Paulo: Manole; 2001. 
7. Catanzariti JF, Salomez E, Bruandet JM, Thevenon A. Visual deficiency and scoliosis. Spine (Phila Pa 1976). 2001 Jan 1;26(1):48-52. http://dx.doi.org/10.1097/00007632-200101010-00010

8. Aulisa L, Bertolini C, Piantelli S, Piazzini DB. Axial deviations of the spine in blind children. Ital J Orthop Traumatol. 1986 Mar;12(1): 85-92.

9. Sanchez HM, Barreto RR, Baraúna MA, Canto RST, Morais EG. Avaliação postural de indivíduos portadores de deficiência visual através da biofotogrametria computadorizada. Fisioter Mov. 2008;21(2):11-20.

10. Simprini R, Braccialli L. Influência do sistema sensório-motor na manutenção da postura estática em indivíduos cegos. Infanto Rev Neuropsiquiatr Infanc Adolesc. 1998;6(1):26-38.

11. Pogrund R, Rosen S. The preschool blind child can be a cane user. J Vis Impair Blind. 1989;83:431-9.

12. Teodori RM, Negri JR, Cruz MC, Marques AP. Reeducação Postural Global: uma revisão da literatura. Braz J Phys Ther. 2011;15:185-9. http://dx.doi.org/10.1590/S1413-35552011000300003

13. Dimitrova E, Rohleva M. Global postural reeducation in the treatment of postural impairments. Res Kinesiology. 2014;4(1):72-5.

14. Lawand P, Lombardi Júnior I, Jones A, Sardim C, Ribeiro LH, Natour J. Effect of a muscle stretching program using the global postural reeducation method for patients with chronic low back pain: A randomized controlled trial. Joint Bone Spine. 2015 Jul;82(4):272-7. http:// dx.doi.org/10.1016/j.jbspin.2015.01.015

15. Barroqueiro C, Morais NV. The effects of a global postural reeducation program on an adolescent handball player with isthmic spondylolisthesis. J Bodyw Mov Ther. 2014 Apr;18(2):244-58. http://dx.doi.org/10.1016/j.jbmt.2013.10.002

16. Toledo PCV, Mello DBd, Araújo ME, Daoud R, Dantas EHM. Efeitos da Reeducação Postural Global em escolares com escoliose. Fisioter Pesqui. 2011;18(4):329-34. http://dx.doi.org/10.1590/S1809-29502011000400006

17. Marques AP. Escoliose tratada com Reeducação Postural Global. Rev Fisioter Univ São Paulo. 1996;3(12):65-8.

18. Segura DCA, Nascimento FCD, Guilherme JH, Sotoriva P. Efeitos da Reeducação Postural Global aplicada em adolescentes com escoliose idiopática não estrutural. Arq Ciências Saúde UNIPAR. 2013;17(3):153-7.

19. Vanti C, Generali A, Ferrari S, Nava T, Tosarelli D, Pillastrini P. La Rieducazione Posturale Globale nelle patologie muscolo-scheletriche: evidenze scientifiche e indicazioni cliniche. Reumatismo. 2007;59(3):192-201.

20. Souchard PE. RPG:Fundamentos da Reeducação Postural Global: princípios e originalidade. São Paulo: É Realizações; 2003.

21. Ovadia D, Bar-On E, Fragnière B, Rigo M, Dickman D, Leitner J, Wientroub S, Dubousset J. Radiation-free quantitative assessment of scoliosis: a multi center prospective study. Eur Spine J. 2007 Jan;16(1):97-105. http://dx.doi.org/10.1007/s00586-006-0118-8

22. Dao T, Labelle H, Le Blanc R. Variabilité intra-observateur de la mesure de la posture à láide dún numérisateur tridimensionnel. Ann Chir. 1997;51(8):848-53.

23. Grivas TB, Savvidou OD, Vasiliadis E, Psarakis S, Koufopoulos G. Prevalence of scoliosis in women with visual deficiency. Stud Health Technol Inform. 2006;123:52-6.

24. Sá CG, Bim CR. Análise estabilométrica pré e pós-exercícios fisioterapêuticos em crianças deficientes visuais. Fisioter Mov. 2012;25: 811-9. http://dx.doi.org/10.1590/S0103-51502012000400014

25. Meereis E, Lemos L, Pranke G, Alves R, Teixeira C, Mota C. Deficiência visual: uma revisão focada no equilíbrio postural, desenvolvimento psicomotor e intervenções. Rev Bras Ci e Mov. 2011;19(1):108-13.

26. Frade MCM, Carde-a JP, Shimano SGN, Oliveira CCES, Oliveira NML. Equilíbrio dos deficientes visuais antes e após gameterapia. Rev Educ Espec. 2014;27(50):751-64. http://dx.doi.org/10.5902/1984686X13720

27. Domholdt E. Physical therapy research: principles and applications. $2^{\mathrm{a}}$ ed. Philadelphia: W.B. Saunders; 2000.

28. Santos A. Diagnóstico clínico postural: um guia prático. $2^{\mathrm{a}}$ ed. São Paulo: Sammus; 2001.

29. Leroux MA, Zabjek K, Simard G, Badeaux J, Coillard C, Rivard CH. A noninvasive anthropometric technique for measuring kyphosis and lordosis: an application for idiopathic scoliosis. Spine. 2000 Jul 1;25(13):1689-94. http://dx.doi.org/10.1097/00007632-20000701000012

30. Vedantam R, Lenke LG, Keeney JA, Bridwell KH. Comparison of standing sagittal spinal alignment in asymptomatic adolescents and adults. Spine. 1998 Jan 15;23(2):211-5. http://dx.doi.org/10.1097/00007632-199801150-00012

31. Bradford D, Lostein J, Moe J, Ogilvie J, RB W. Escoliose e outras deformidades da coluna "O livro de Moe”. São Paulo: Santos; 1994.

32. Rosário JLP, Sousa A, Cabral CMN, João SMA, Marques AP. Reeducação postural global e alongamento estático segmentar na melhora da flexibilidade, força muscular e amplitude de movimento: um estudo comparativo. Fisioter Pesq. 2008; 15(1):12-8. http://dx.doi.org/10.1590/ S1809-29502008000100003

33. Fregonesi CEPT, Valsechi CM, Masselli MR, Faria CRS, Ferreira DMA. Um ano de evolução da escoliose com RPG. Fisioter Bras. 2007;8(2):140-2.

34. Rossi LP, Brandalize M, Gomes ARS. Efeito agudo da técnica de reeducação postural global na postura de mulheres com encurtamento da cadeia muscular anterior. Fisioter Mov. 2011;4(2):255-63. http://dx.doi.org/10.1590/S0103-51502011000200007

35. Bettany-Saltikov J, Parent E, Romano M, Villagrasa M, Negrini S. Physiotherapeutic scoliosis-specific exercises for adolescents with idiopathic scoliosis. Eur J Phys Rehabil Med. 2014 Feb;50(1):111-21.

36. Morningstar MW, Woggon D, Lawrence G. Scoliosis treatment using a combination of manipulative and rehabilitative therapy: a retrospective case series. BMC Musculoskelet Disord. 2004 Sept 14;5:32. http://dx.doi.org/10.1186/1471-2474-5-32 
37. Ferguson LW. Adult Idiopathic scoliosis: The tethered spine. J Bodyw Mov Ther. 2014 Jan;18(1):99-111. http://dx.doi.org/10.1016/j. jbmt.2013.05.002

38. Hebela NM, Tortolani PJ. Idiopathic scoliosis in adults: classification, indications, and treatment options. Semin Spine Surg. 2009;21(1): 16-23. http://dx.doi.org/10.1053/j.semss.2008.11.003

39. Meereis E, Lemos L, Pranke G, Alves R, Teixeira C, Mota C. Deficiência visual: uma revisão focada no equilíbrio postural, desenvolvimento psicomotor e intervenções. Rev Bras Ci e Mov. 2011;19(1):108-13.

40. Cardoso L, Gonçalves C, Bonvicine C, Barboza M. Análise clínica e radiográfica pré e pós-tratamento conservador na escoliose idiopática do adolescente: estudo de caso. ConScientiae Saúde. 2011;10(1):166-74.

41. Gauchard GC, Lascombes P, Kuhnast M, Perrin PP. Influence of different types of progressive idiopathic scoliosis on static and dynamic postural control. Spine. 2001 May 1;26(9):1052-8. http://dx.doi.org/10.1097/00007632-200105010-00014

42. Isableu B, Ohlmann T, Cremieux J, Amblard B. Selection of spatial frame of reference and postural control variability. Exp Brain Res. 1997 May;114(3):584-9. http://dx.doi.org/10.1007/PL00005667

43. Patla AE. Understanding the roles of vision in the control of human locomotion. Gait Posture. 1997;5(1):54-69. http://dx.doi.org/10.1016/ S0966-6362(96)01109-5

44. Moseley GL, Hodges PW. Reduced variability of postural strategy prevents normalization of motor changes induced by back pain: a risk factor for chronic trouble? Behav Neurosci. 2006 Apr;120(2):474-6. http://dx.doi.org/10.1037/0735-7044.120.2.474

45. Durkin JL, Harvey A, Hughson RL, Callaghan JP. The effects of lumbar massage on muscle fatigue, muscle oxygenation, low back discomfort, and driver performance during prolonged driving. Ergonomics. 2006 Jan 15;49(1):28-44. http://dx.doi.org/10.1080/ 00140130500356882 